\title{
НОВЫЙ ПОДХОД К ЛЕЧЕНИЮ АНЕВРИЗМЫ БРЮШНОЙ АОРТЫ
}

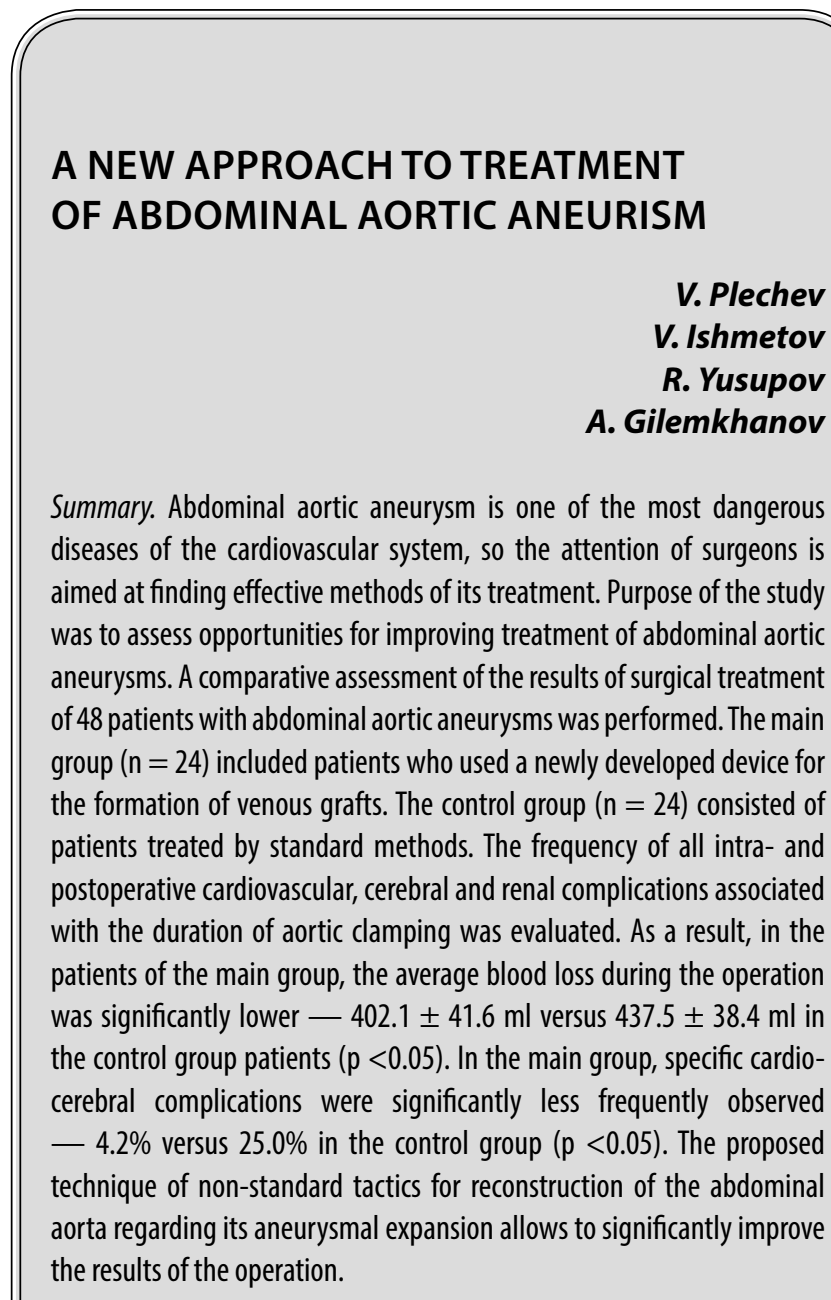

Keywords: abdominal aortic aneurysm, resection, prosthetics, complications.

\section{Актуальность}

A невризма брюшной аорты - локальное увеличение брюшной аорты, при котором диаметр сосуда увеличивается от 3 и более см. С каждым годом объем аневризматического мешка вырастает на $10 \%$, в результате чего без своевременного лечения происходит истончение и разрыв аневризмы [3]. Подобные клинические ситуации непосредственно угрожают жизни при отсутствии экстренной хирургической помощи или возможных в настоящее время альтернативных миниинвазивных интервенционных эндовакулярных манипуляций [2].
Плечев Владимир Вячеславович

Профессор, Башкирский государственный медицинский университет

Ииметов Владимир Шамилевич

Профессор, Башкирский государственный медицинский университет Юсупов Ринат Хабибович

Аспирант, Башкирский государственный медицинский университет Yusupov_80@mail.ru

Гилемханов Альберт Радикович

Аспирант, Башкирский государственный медицинский университет

Аннотация. Аневризма брюшной аорты является одним из наиболее опасных заболеваний сердечно-сосудистой системы, поэтому внимание хирургов направлено на поиск эффективных методов её лечения. Целью исследования стала оценка возможности улучшения лечения аневризмы брюшной аорты. Проведена сравнительная оценка результатов хирургического лечения 48 пациентов с аневризмами брюшной аорты. В основную группу $(\mathrm{n}=24)$ вошли пациенты, у которых использовалось новое разработанное устройство для формирования венозных графтов. Контрольную группу $(\mathrm{n}=24)$ составили пациенты, лечившиеся по стандартной методике. Оценивалась частота всех интра- и послеоперационных сердечно-сосудистых, мозговых и почечных осложнений, связанных с длительностью пережатия аорты. В результате отмечено, что у пациентов основной группы достоверно ниже был средний объем кровопотери во время операции - 402,1 141,6 мл против 437,5 $\pm 38,4$ мл у пациентов контрольной группы ( $<<0,05)$. Так же в основной группе достоверно реже наблюдались специфические карди0-церебральные осложнения - 4,2\% против 25,0\% в группе контроля $(p<0,05)$. Таким образом, предложенная методика нестандартной тактики реконструкции брюшной аорты по поводу ее аневризматического расширения позволяет достоверно улучшить результаты операции.

Ключевые слова: аневризма брюшной аорты, резекция, протезирование, осложнения.

В связи с неблагоприятным прогнозом, хирургическое лечение аневризматических изменений брюшной аорты весьма актуально.

Впервые эндоваскулярные реконструкции аневризмы инфраренального отдела брюшной аорты у человека были выполнены еще в 1991 году. На сегодняшний день в Российской Федерации отмечается устойчивая тенденция к увеличению количества эндоваскулярных вмешательств при аневризмах брюшного отдела аорты, поскольку данный метод является оптимальным. При имплантации графтов вмешательство осуществляется под местным обезболиванием, кровопотеря в ходе опе- 

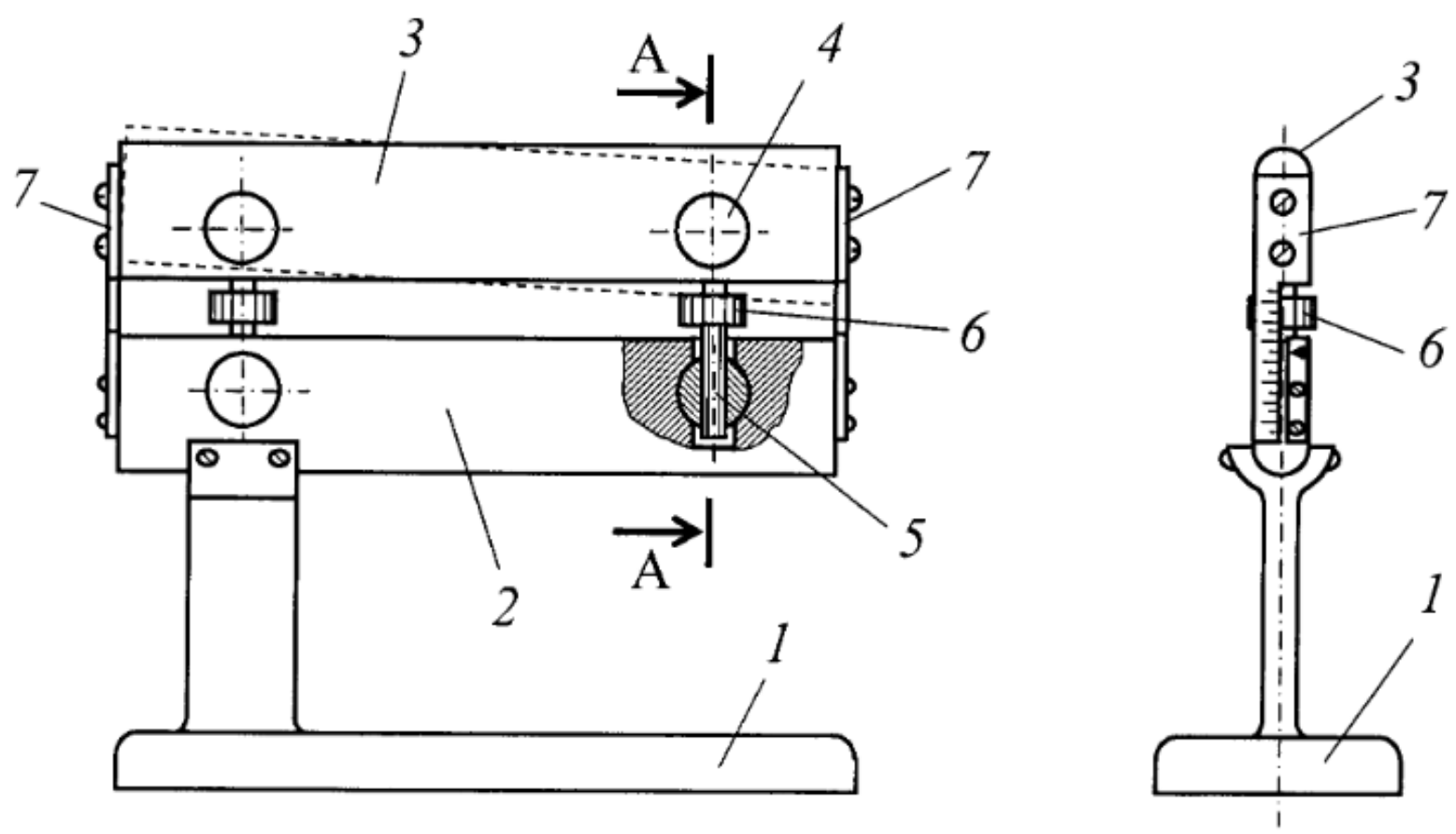

Рис. 1. Схема устройства

1 - корпус, 2 - нижняя пластина матрицы; 3 - верхняя пластина матрицы; 4 - резьбовая втулка; 5 - винтовая стойка с правой и левой резьбой; 6 - рифленый регулировочный ролик; 7 - шкала указания диаметра формируемого графта.

рации минимальна, отсутствует необходимость в пережатии брюшной аорты, что сводит к минимуму возникновение возможных интра-и послеоперационных осложнений со стороны сердечно-сосудистой системы, к тому же значительно сокращается продолжительность послеоперационного периода $[1,4,11]$.

В 1974 г. была опубликована статья, в которой впервые была описана техника формирования спиральных венозных графтов, в клинической практике первый раз она была применена в 1976 г. D.B. Doty, W.H. Baker и др. для лечения пациента с синдромом верхней полой вены [8]. Уже в XXI веке появились данные об успешном применении спиральных венозных графтов для протезирования брюшной аорты, они позволяют достичь точного соответствия диаметров кондуита и протезируемого сосуда, благодаря чему достигаются гораздо лучшие результаты лечения по сравнению с синтетическими протезами $[5,7,12]$.

Несмотря на все преимущества описываемой методики, существуют некоторые проблемы, требующие решения, в частности, внимание исследователей направлено на расширение возможностей инструмента для выполнения графтов любого диаметра, повышение удобства использования инструментов, и, как результат, снижение интра- и послеоперационных осложнений.

\section{Це^ь}

Оценить возможности улучшения лечения аневризмы брюшной аорты.

\section{Материалы и метомы}

Под наблюдением находились 48 пациентов с аневризмами инфраренального отдела аорты, лечившихся в Клинике Башкирского государственного медицинского университета в период с 2016 по 2019 гг. В зависимости от методов хирургической коррекции они были разделены на 2 группы. В основную группу вошли 24 пациента, оперированные по разработанной в клинике методике. Согласно данной методике для формирования венозных графтов использовалось специальное устройство (Патент на полезную модель — RU183859 U1 от 19.03.2018).

Устройство для формирования венозных графтов представляет собой корпус с закрепленной на нем матрицей, которая содержит две пластины с закругленными ребрами, установленные в одной плоскости и соединенные винтовыми стойками, содержащими участки с правой и левой резьбой, а торцы пластин снабжены указателями со шкалой диаметров формируемых графтов. Благодаря такой конструкции обеспечивается возможность формирования как цилиндрических, так и конусовидных 
Таблица 1. Характеристика исследуемых групп

\begin{tabular}{|l|l|l|l|}
\hline Показатели & Основная группа & Группа контроля & $\mathbf{p}$ \\
\hline Количество мужчин (абс.,\%) & $18(75,0)$ & $16(66,6)$ & $>0,05$ \\
\hline Средний возраст (лет) & $64,9 \pm 6,2$ & $65,6 \pm 5,6$ & $>0,05$ \\
\hline Средний диаметр проксимальной части аневризмы (мм) & $51,3 \pm 3,5$ & $51,2 \pm 3,8$ & $>0,05$ \\
\hline Средний диаметр максимально расширенной части аневризмы (мм) & $64,7 \pm 5,1$ & $64,1 \pm 4,2$ & $10,4 \pm 5,1$ \\
\hline Средняя длина аневризмы (мм) & $10,9 \pm 4,6$ & $14(58,3)$ & $>0,05$ \\
\hline Ишемическая болезнь сердца (абс.,\%) & $19(79,2)$ & $3(12,5)$ & $>0,05$ \\
\hline Инфаркт в анамнезе (абс.,\%) & $6(25,0)$ & $2(8,3)$ & $>0,05$ \\
\hline Острое нарушение мозгового кровообращения в анамнезе (абс.,\%) & $5(20,8)$ & $4(16,7)$ & $>0,05$ \\
\hline Сахарный диабет (абс.,\%) & $9(37,5)$ & $>0,05$ \\
\hline
\end{tabular}

Таблица 2. Характеристика исследуемых групп. Сравнительная оценка некоторых операционных показателей у пациентов исследуемых групп.

Сравниваемые параметры
\begin{tabular}{|l|l|l|l|}
\hline Продолжительность операции (мин.) & Основная группа & Контрольная группа & $\mathbf{p}$ \\
\hline Средняя продолжительность пережатия аорты (мин.) & $259,1 \pm 14,7$ & $256,2 \pm 12,4$ & $>0,05$ \\
\hline $\begin{array}{l}\text { Среднее время накладывания проксимального анастомоза } \\
\text { (мин.) }\end{array}$ & $12,1 \pm 0,9$ & $20,2 \pm 1,4$ & $>0,05$ \\
\hline $\begin{array}{l}\text { Среднее время накладывания первого дистального анастомоза } \\
\text { (мин.) }\end{array}$ & $11,4 \pm 0,8$ & $12,3 \pm 1,1$ & $>0,05$ \\
\hline Средний объем кровопотери (мл) & $402,1 \pm 41,6$ & $11,2 \pm 0,7$ & $>0,05$ \\
\hline АД после пережатия аорты (мм рт. ст.) & $152,4 \pm 14,1$ & $153,1 \pm 14,7$ & $<0,05$ \\
\hline
\end{tabular}

Таблица 3. Послеоперационные результаты исследуемых групп

\begin{tabular}{|l|l|l|l|}
\hline Послеоперационные показатели & Основная группа & Контрольная группа & $\mathbf{p}$ \\
\hline Специфические кардио-церебральные осложнения (абс.,\%) & $1(4,2)$ & $6(25,0)$ & $<0,05$ \\
\hline Неспецифические осложнения (абс.,\%) & $2(8,2)$ & $6(25)$ & $>0,05$ \\
\hline Летальные исходы (абс.,\%) & $1(4,2)$ & $3(12,5)$ & $>0,05$ \\
\hline
\end{tabular}

спиральных венозных графтов диаметром от 15 до 25 мм с бесступенчатым изменением размеров, что соответствует диаметрам крупных сосудов (аорте, крупным магистральным артериям, верхней и нижней полой вене). Также устройство обладает простой конструкцией, позволяющей без затруднений осуществить его сборку и разборку, что упрощает и повышает качество стерилизации.

На рисунке 1 представлена схема устройства.

В контрольную группу вошли 24 пациента, при оперировании которых использовались синтетические протезы.
Обе группы были сопоставимы по полу, возрасту, сопутствующей патологии, локализации и размерам аневризм. Более подробно характеристика групп представлена в таблице 1.

Всем пациентам выполнялись необходимые дополнительные методы исследования согласно клиническим протоколам по ведению пациентов, страдающих аневризмой брюшной аорты.

Для изучения преимуществ и недостатков разработанной методики проводилась сравнительная оценка интра- и послеоперационных показателей. 
Статистическая обработка полученных данных была проведена с использованием программ STATISTICA v.10.0. Данные представлены в виде средних значений и стандартных отклонений для непрерывных переменных. Для сравнения количественных данных применяли t критерии Стьюдента, качественные данные оценивались с использованием Хи-квадрата. Достоверными считались результаты при $p<0,05$.

\section{Результаты}

Общая продолжительность операции у пациентов обеих групп были сопоставимыми. Время пережатия аорты так же не отличалось. Не отмечено достоверной разницы при накладывании проксимального анастомоза и уровня АД после пережатия аорты, и пуска кровотока у больных обеих групп (таблица 2).

Обращает внимание разница в объёме кровопотери, который в основной группе был более чем на 30 мл меньше по сравнению с группой контроля. Полученные различия объясняются точным сопоставлением протезируемого сосуда с протезом в области проксимального и дистального анастомоза.

Непосредственные послеоперационные результаты представлены в таблице 3.

Согласно полученным данным, во всех случаях отмечалось достоверно меньшее число специфических осложнений у пациентов, оперированных по разработанной методике.

Частота таких неспецифических осложнений, как нагноение раны $(n=4)$ и нарушения мочеиспускания $(n=2)$ была так же выше (p>0,05) у пациентов контрольной группы, однако различия не достигли уровня статистической значимости.

Как видно из таблицы, среди пациентов основной группы в 1 (4,2\%) случае развились кардио- церебральные осложнения в виде геморрагического инсульта $(n=1)$, который послужил причиной летального исхода. Летальные исходы, обусловленные инфарктом миокарда и инсультом, у пациентов контрольной группы были зарегистрированы в 3 (12,5\%) случаях (р>0,05).

Выжившие пациенты были выписаны из стационара в сроки от 8 до 19 дней после операции с последующим диспансерным наблюдением.

В отдаленном периоде у пациентов обеих групп случаи летальных исходов не зарегистрированы. Все 44 пациента (23 из основной и 21 из контрольной групп) на момент завершения исследования находились под контролем кардиолога и сосудистого хирурга и вели обычный образ жизни.

\section{Обсужление}

Одной из главных задач при реконструкции брюшной аорты по поводу аневризмы становится максимальное уменьшение факторов, влияющих на развитие осложнений и летальных исходов. Значительных успехов в данном направлении удалось достичь благодаря использованию трансплантатов, изготовленных из собственных тканей пациента. Использование техники формирования спиральных венозных графтов, позволяет достичь точного соответствия диаметров протеза и протезируемого сосуда, что в дальнейшем существенно снижает количество осложнений и улучшает прогноз пациента [7].

В качестве матрицы для формирования спиральных венозных графтов предлагалось использовать различные приспособления: двухпросветную венозную канюлю для аппарата искусственного кровообращения, торакальную дренажную трубку с наружным диаметром от 6,7 до 11,3 мм, бужи (расширители, дилататоры) Гегара $[8,10]$.

Однако все вышеперечисленные приспособления имеют ряд недостатков. Во-первых, диаметр крупных сосудов, таких как аорта, верхняя и нижняя полые вены, в норме может достигать 25 мм, и ни одно из этих приспособлений не позволяет сформировать спиральный венозный графт такого диаметра. Диаметр формируемого на этих аналогах графта может не соответствовать протезируемому сосуду, что повышает вероятность стеноза в области анастомоза и, как следствие, приводит к ишемии дистально расположенных органов и тканей. Во-вторых, при протезировании большого участка сосуда предпочтительней использовать конусовидный протез для более точного сопоставления протезируемого сосуда с протезом в области проксимального и дистального анастомоза, что невозможно при использовании перечисленных приспособлений. При формировании цилиндрического графта диаметром, равным проксимальному концу сосуда, в области дистального анастомоза неизбежен избыток диаметра спиральной конструкции, в результате чего при сшивании двух концов образуются фестоны и карманы, через которые возможно подтекание крови и образование пристеночных тромбов.

В проведенном исследовании количество перии пост-операционных осложнений при использовании предложенной методики минимально. Соответственно в дальнейшем, мы можем прогнозировать снижение риска развития стенозов, тромбозов и прочих интраи послеоперационных осложнений для пациента. Также устройство обладает простой конструкцией, позволяющей без затруднений осуществить его сборку и разбор- 
ку, что упрощает и повышает качество стерилизации, минимизируя риск инфекционных осложнений.

\section{Зак^ючение}

C помощью предложенной методики возможно сформировать индивидуальные сосудистые протезы из собственной венозной ткани больного диаметром, точно соответствующим протезируемому сегменту, обладающие резистентностью к инфекции. Техника проста, легко воспроизводима, однако, для того чтобы определить место предложенного инструмента в реальной клинической практике, необходимо провести дополнительные экспериментальные исследования.

\section{ЛИТЕРАТУРА}

1. Алекян Б.Г., Григорьян А. М., Стаферов А. В. Состояние рентгенэндоваскулярной диагностики и лечения в Российской Федерации (2016 г.). Эндоваскулярная хирургия. 2017; 4 (2): 75-92.

2. Ашер э. Сосудистая хирургия по Хаймовичу. М.: ОАО «Издательство «БИНОМ.Лаборатория знаний». 2012; 531.

3. Каплан М.Л., Андреева Д. Д., Фолитарик В. И. Аневризма брюшной аорты и ее осложнения. Синергия наук. 2017; 10:895-899.

4. Кокоев В.Г., Сасина Е. В., Поляк М. И. и др. Имплантация стент-графта при аневризме брюшного отдела аорты. Главный врач. 2015; 3 (45):36-39.

5. Aerts P.D., van Zitteren M., Van Kasteren M. E. et al. Report of two in situ reconstructions with a saphenous spiral vein graft of Coxiella burnetii-infected aneurysms of the abdominal aorta. J. Vasc. Surg. 2013; 57(1):234-7.

6. Chiu K.M., Chu S. H., Chen J.S. et al. Spiral saphenous vein graft for portal vein re-construction in pancreatic cancer surgery. Vasc. Endovascular. Surg. 2007; 41(2):149-52.

7. Chiu C.J., Terzis J., MacRae M. L. Replacement of superior vena cava with the spiral composite vein graft. A versatile technique. Ann Thorac Surg. 1974; 17(6):555-60

8. Doty D.B., BakerW.H. Bypass of superior vena cava with spiral vein graft. Ann Thorac. Surg. 1976. Nov.; 22(5):490-3.

9. Hagino R.T., Bengston T. D., Fosdick D. A. et al. Venous reconstructions using the superficial femoralpopliteal vein. J. Vasc. Surg. 1997; 26: 829-837.

10. Koga Y., Tomita M., Shibata K. et al. An experience using spiral vein graft as arterial substitute. Jpn. J. Surg. 1981; 11:305-9.

11. Neufang A., Savvidis S. Operative technique and morbidity of superficial femoral vein harvest. Gefasschirurgie. 2016; 21 (Suppl. 2): 45-54.

12. van Zitteren M., van der Steenhoven T. J., Burger D. H. et al. Spiral vein reconstruction of the infected abdominal aorta using the greater saphenous vein: preliminary results of the Tilburg experience. Eur. J. Vasc. Endovasc. Surg. 2011; 41(5):637-46. 